\title{
Temperature Dependence of Magnetization Damping in Heusler Alloy Films
}

\author{
J. Dubowik, M. ZaŁęski, H. GŁowiński \\ Institute of Molecular Physics, Polish Academy of Sciences, M. Smoluchowskiego 17, 60-179 Poznań, Poland \\ AND I. GościańsKA \\ Department of Physics, A. Mickiewicz University, Umultowska 85, 61-614 Poznań, Poland \\ The Gilbert damping for $\mathrm{Co}_{2} \mathrm{CrAl}\left(\mathrm{Co}_{2} \mathrm{Cr}_{0.3} \mathrm{Fe}_{0.7} \mathrm{Al}\right)$ and $\mathrm{Ni}_{2} \mathrm{MnSn}$ Heusler alloy films was investigated as a \\ function of temperature. The best films reveals the Gilbert damping nearly independent of temperature. Magnetic \\ relaxation in $\mathrm{Co}_{2} \mathrm{CrAl}$ is strongly influenced by magnetic inhomogeneities due to phase separation. The best \\ $\mathrm{Ni}_{2} \mathrm{MnSn}$ epitaxial films have comparable Gilbert damping $\alpha$ of $6 \times 10^{-3}$ as half-metallic $\mathrm{Co}_{2} \mathrm{Cr}_{0.3} \mathrm{Fe}_{0.7} \mathrm{Al}$ films \\ with $\alpha=3 \times 10^{-3}$.
}

PACS numbers: 76.50.+g, 75.70.Ak, 75.78.Jp

\section{Introduction}

It has been recently shown, both theoretically [1] and experimentally $[2,3]$, that the Gilbert damping constant $\alpha$ in half-metallic (HM) Co-based full Heusler alloys (HA) is low. Magnetization damping is directly related to viscous-damping-like term in the Landau-LifshitzGilbert equation $(\alpha / M)(\boldsymbol{M} \times \mathrm{d} \boldsymbol{M} / \mathrm{d} t) / M$ and described by the ferromagnetic resonance (FMR) linewidth $\Delta H$ (peak-to-peak of the first derivative of the FMR absorption) $\Delta H=2 / \sqrt{3} \alpha(\omega / \gamma)$, where $\omega$ is the angular frequency of microwaves and $\gamma$ is the gyromagnetic ratio. In the case of thin ferromagnetic films there are various extrinsic and intrinsic contributions to the linewidth and, in fact, the measured $\Delta H$ in the perpendicular configuration (i.e. with the field applied perpendicular to the film plane) is believed to be given exactly by the Gilbert damping [4]. If no additional line broadening comes from magnetic inhomogeneities (such as local resonances) in the parallel configuration extrinsic damping due to two-magnon scattering plays an important role [4].

The Gilbert damping constant $\alpha$ in some HM HA films can be as low as 0.003 in $\mathrm{Co}_{2} \mathrm{Fe}_{0.4} \mathrm{Mn}_{0.6} \mathrm{Si}$ [2] or even 0.001 in $\mathrm{Co}_{2} \mathrm{FeAl}$ [3] which gives the relaxation frequency $G=\alpha \gamma M_{\mathrm{S}}$ of 50 and $20 \mathrm{MHz}$, respectively. This makes HA attractive for low damping materials for future spintronic applications [1]. However little is known on temperature dependence of magnetization damping in HA films. A theoretically predicted dependence for the damping constant on the electrical resistivity of HA reveals minimum as a function of temperature due to resistivity-like and conductivity-like contributions [1].

In this contribution we present temperature dependence of FMR linewidth of $\mathrm{Co}_{2} \mathrm{CrAl}$ (and
$\mathrm{Co}_{2} \mathrm{Cr}_{0.3} \mathrm{Fe}_{0.7} \mathrm{Al}$, too) and compare it to that of $\mathrm{Ni}_{2} \mathrm{MnSn} \mathrm{HA}$ films in order to find the influence of half-metallicity. $\quad \mathrm{Co}_{2} \mathrm{CrAl}$ is theoretically predicted half-metal HA with a band-gap in the minority spin band of $0.8 \mathrm{eV}[5] . \mathrm{Ni}_{2} \mathrm{MnSn}$ reveals no $\mathrm{HM}$ properties (i.e. it has no band-gap [6]) but both HA have almost the same Curie temperature of $320 \mathrm{~K}$ and $330-350 \mathrm{~K}$, respectively.

\section{Experimental details and discussion}

The $\mathrm{Co}_{2} \mathrm{CrAl}$ films were prepared by using magnetron sputtering system $\left(p_{\text {base }}<2 \times 10^{-8}\right.$ Torr $)$ onto Si (100) oxidized substrates. $\mathrm{Ni}_{2} \mathrm{MnSn}$ films were prepared by either flash evaporation method (polycrystalline films) or by sputtering onto $\mathrm{MgO}(100)$ substrates at $300-350{ }^{\circ} \mathrm{C}$ in order to achieve proper structural ordering. The crystalline ordering of $\mathrm{Co}_{2} \mathrm{CrAl}$ and $\mathrm{Ni}_{2} \mathrm{MnSn}$ films was $\mathrm{B} 2$ and $\mathrm{L} 2{ }_{1}$, respectively, as confirmed by X-ray diffraction (XRD) and transmission electron microscopy (TEM). Thickness of the film varied between 12 and $180 \mathrm{~nm}$. Ferromagnetic resonance was measured with a standard Xband spectrometer $(9.2 \mathrm{GHz})$ in a temperature range between $130 \mathrm{~K}$ (4.2 for $\left.\mathrm{Co}_{2} \mathrm{CrAl}\right)$ and $350 \mathrm{~K}$. Since quantitative measurements of the actual site ordering by XRD is difficult, we measured the effective magnetization of the samples to compare it with the magnetization of the best ordered bulk samples. Extrapolated to $0 \mathrm{~K}$ was $440 \mathrm{G}$ $\left(2.4 \mu_{\mathrm{B}} /\right.$ f.u. $)$ and $637 \mathrm{G}\left(3.6 \mu_{\mathrm{B}} /\right.$ f.u. $)$ for $\mathrm{Co}_{2} \mathrm{CrAl}$ and $\mathrm{Ni}_{2} \mathrm{MnSn}$, respectively. These results are $10-20 \%$ lower than that reported for the best ordered bulk samples (3 and $4 \mu_{\mathrm{B}} /$ f.u.) $[7,8]$. The Gilbert damping $\alpha$ was determined from $\Delta H$ measured at the perpendicular configuration. By independent measurements of $\Delta H$ as a 
function of magnetization angle from the film normal at room temperature we checked that when the magnetization is inclined to the film plane the linewidth increases by about 100-200 Oe in accordance with two-magnon scattering picture [4].

Figure 1 shows temperature dependence of $\Delta H^{\perp}$ and $\Delta H^{\|}$of two $\mathrm{Ni}_{2} \mathrm{MnSn}$ films measured at perpendicular and parallel configurations, respectively. For the polycrystalline film (full dots and squares), it is seen that for both configurations $\Delta H$ is nearly independent of temperature in the range of $250-300 \mathrm{~K}$ (just below the Curie temperature of $330-350 \mathrm{~K}$ ) and monotonically increases as temperature is lowered to $100 \mathrm{~K}$. However the difference between $\Delta H^{\perp}$ and $\Delta H^{\|}$increases substantially with decreasing temperature. For the epitaxial film (open circles and squares) the trend is generally the same but $\Delta H$ for both configurations is much lower, especially for the perpendicular configuration. In order to give evidence for two-magnon scattering we additionally investigated the FMR linewidth of the epitaxial film as a function of the angle between magnetization and the normal (not shown) and checked that $\Delta H^{\perp}$ is mainly given by the intrinsic Gilbert damping with $\Delta H^{\perp}$ as low as 20 Oe at $250 \mathrm{~K}$. The Gilbert damping coefficient $\alpha$ is of 0.006 .

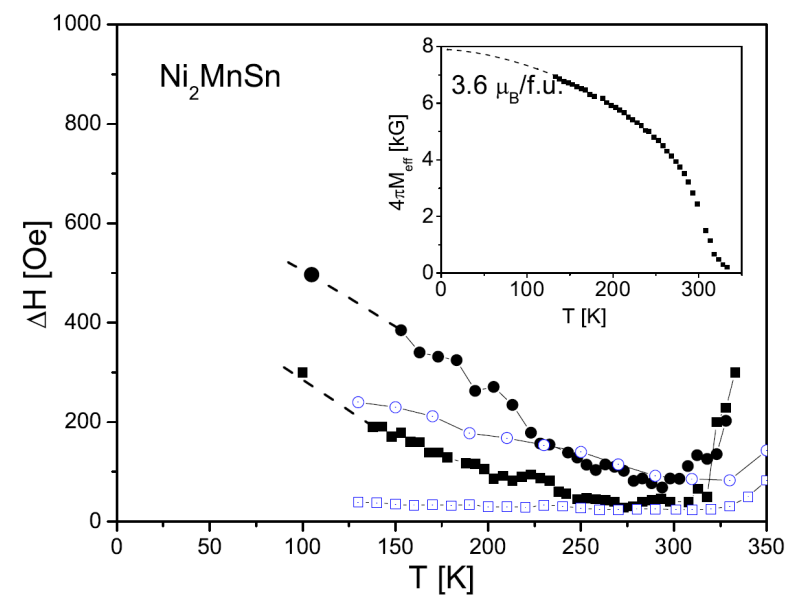

Fig. 1. Temperature dependence of FMR linewidth of $\mathrm{Ni}_{2} \mathrm{MnSn}$ films measured at perpendicular (full and open squares) and parallel (full and open circles) configurations. Inset shows temperature dependence of the effective magnetization.

Figure 2 shows the temperature dependence of $\Delta H^{\perp}$ and $\Delta H^{\|}$of $\mathrm{Co}_{2} \mathrm{CrAl}$ film. $\Delta H^{\perp}$ decreases with decreasing temperature and attains minimal value of 108 Oe at $140 \mathrm{~K}$ and then unexpectedly grows up to 3700 Oe at $4 \mathrm{~K}$. $\Delta H^{\|}$changes in a similar way but broadening in the linewidth onsets at temperatures lower than $200 \mathrm{~K}$. We interpret such a behavior as due to strong magnetic inhomogeneities of polycrystalline $\mathrm{Co}_{2} \mathrm{CrAl}$ with $\mathrm{B} 2$ structure. Indeed, in a temperature region of $100-250 \mathrm{~K}$ we observed clear resolved spin wave spectra (SWR) that however display separation independent of mode number. This implies a depth-dependent magnetization profile in- side the film in accordance to the Portis model of volume inhomogeneity [9]. We interpret the strong broadening in $\Delta H$ and SWR as resulting from inclusion of $\mathrm{CoCr}$ phase in our $\mathrm{Co}_{2} \mathrm{CrAl}$ film. Presumably $\mathrm{Co}-\mathrm{Cr}$ antisite disorder favors formation of CoCr phase with 20-25 at.\% of $\mathrm{Cr}$ that becomes ferromagnetic at $T<100 \mathrm{~K}$ [10] and results in line broadening. Therefore, magnetization relaxation at low temperature is not intrinsic. If for $\mathrm{Co}_{2} \mathrm{CrAl} \Delta H^{\perp}$ value of about 100 Oe is assumed to be solely due to the Gilbert damping, $\alpha=3 \times 10^{-2}(G=240 \mathrm{MHz})$ is substantially higher than that in the best HM HA [2].

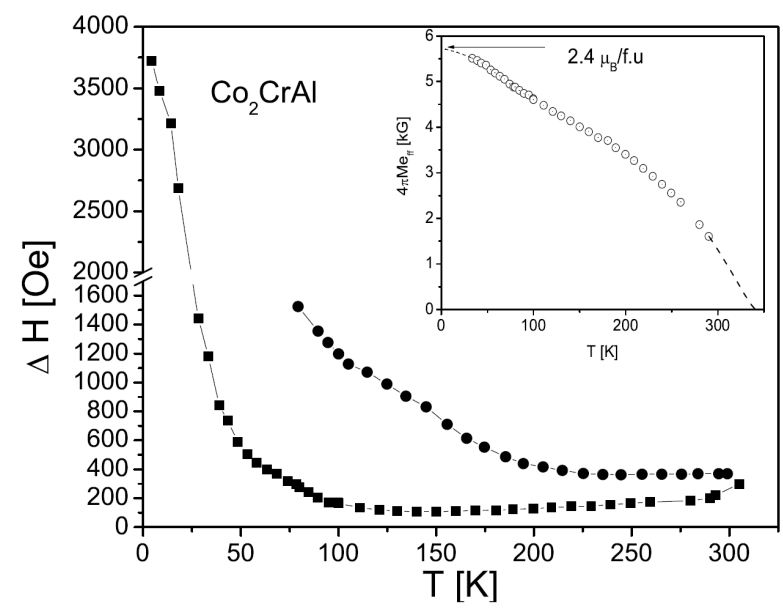

Fig. 2. Temperature dependence of FMR linewidth of $\mathrm{Co}_{2} \mathrm{CrAl}$ film measured at perpendicular (squares) and parallel (circles) configurations. Inset shows temperature dependence of the effective magnetization.

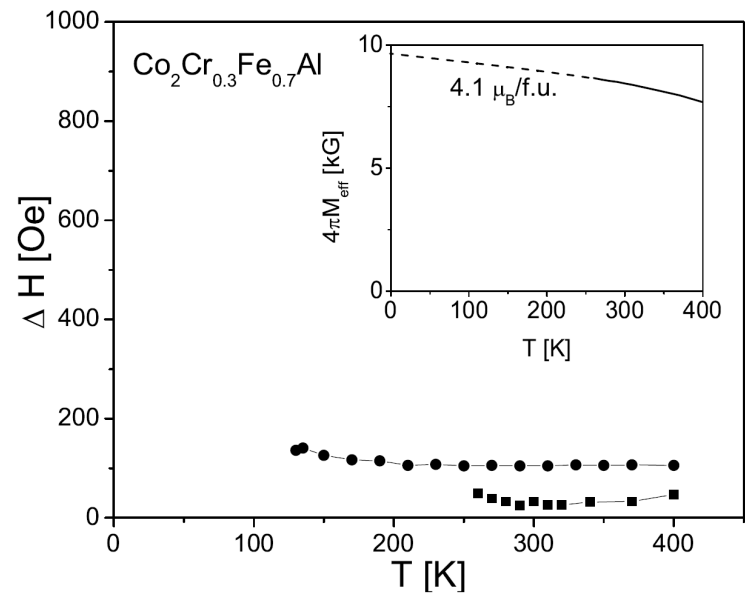

Fig. 3. Temperature dependence of FMR linewidth of $\mathrm{Co}_{2} \mathrm{Cr}_{0.3} \mathrm{Fe}_{0.7} \mathrm{Al}$ film measured at perpendicular (squares) and parallel (circles) configurations. Inset shows temperature dependence of the effective magnetization.

Some $\mathrm{Fe}$ containing $\mathrm{Co}_{2} \mathrm{Cr}_{x} \mathrm{Fe}_{1-x} \mathrm{Al} \mathrm{HA}$ are considered as HM with a high spin polarization [5]. Therefore we find it worthwhile to compare temperature de- 
pendence of $\mathrm{Co}_{2} \mathrm{Cr}_{0.3} \mathrm{Fe}_{0.7} \mathrm{Al}$ film with that of $\mathrm{Co}_{2} \mathrm{CrAl}$. Figure 3 shows a temperature dependence of $\Delta H^{\|}$and $\Delta H^{\perp}$. Since the available field in our electromagnet was too low to observe FMR in perpendicular configuration at $T<250 \mathrm{~K}, \Delta H^{\perp}$ is shown in a relatively narrow temperature region of $250-400 \mathrm{~K}$. One can see that $\Delta H$ for both configurations is nearly independent of temperature with a small increase at the lowest temperatures. The lowest value of $\Delta H^{\perp}$ of 24 Oe at $300 \mathrm{~K}$ is comparable with that of the best HM HA [2]. The respective value of $\alpha$ is of $3 \times 10^{-3}(G=50 \mathrm{MHz})$. These results show that the best ordered $\mathrm{Co}_{2} \mathrm{Cr}_{x} \mathrm{Fe}_{1-x} \mathrm{Al} \mathrm{HA}$ belong to a class of materials with a low Gilbert damping.

\section{Conclusions}

In summary, we investigated temperature dependences of FMR linewidth and confirmed almost temperature independent Gilbert damping constant $\alpha$ from $\Delta H^{\perp}$ vs. T. The values of $\alpha$ for the best $\mathrm{Ni}_{2} \mathrm{MnSn}$ and $\mathrm{Co}_{2} \mathrm{Cr}_{0.3} \mathrm{Fe}_{0.7} \mathrm{Al}$ films were small (of $6 \times 10^{-3}$ and $3 \times 10^{-3}$, respectively) and comparable to that of epitaxial $\mathrm{Co}_{2} \mathrm{Fe}_{x} \mathrm{Mn}_{1-x} \mathrm{Si}[2]$. Since $\mathrm{Ni}_{2} \mathrm{MnSn}$ is not half-metal, our results indicate that the Gilbert damping is mainly determined by density of states of $d$-band in accordance with theoretical calculations [11].

\section{References}

[1] C. Liu, C.K. Mewes, M. Chisiev, T. Mewes, W.H. Butler, Appl. Phys. Lett. 95, 022509 (2009).

[2] T. Kubota, S. Tsunegi, M. Oogane, S. Mizukami, T. Miyazaki, H. Naganuma, Y. Ando, Appl. Phys. Lett. 94, 122504 (2009).

[3] S. Mizukami, D. Watanabe, Y. Ando, Y. Miura, M. Shiraki, T. Miyazaki, J. Appl. Phys. 105, 07 D306 (2009).

[4] B. Heinrich, in: Ultrathin Magnetic Structures, Eds. J.A.C. Bland, B. Heinrich, Vol. 3, Springer, New York 2005.

[5] Y. Miura, K. Nagao, M. Shirai, Phys. Rev. B 69, 144413 (2004).

[6] E. Sasioglu, L.M. Sandratskii, P. Bruno, Phys. Rev. B 71, 214412 (2005).

[7] R. Kelekar, B.M. Clemens, J. Appl. Phys. 96, 540 (2004).

[8] P.J. Webster, K.R.A. Ziebeck, S.L. Town, M.S. Peak, Philos. Mag. B 49, 295 (1984).

[9] A.M. Portis, Appl. Phys. Lett. 2, 69 (1963).

[10] K. Adachi, in: Landolt-Bornstein, New Series III/19a, Springer, Berlin 1986.

[11] V. Kamberský, Phys. Rev. B 76, 134416 (2007). 